\title{
BMJ Open Study protocol for a randomised controlled trial of consenting processes and their effects on patient decision- making when undergoing spinal injections: the Risks In Spinal Consenting for Surgery (RISCS) trial
}

James W A Fletcher, ${ }^{1,2}$ Mohsin Khan,, ${ }^{2,3}$ Paul L P J Thorpe ${ }^{4}$

To cite: Fletcher JWA, Khan M, Thorpe PLPJ. Study protocol for a randomised controlled trial of consenting processes and their effects on patient decision-making when undergoing spinal injections: the Risks In Spinal Consenting for Surgery (RISCS) trial. BMJ Open 2018;8:e023426. doi:10.1136/ bmjopen-2018-023426

- Prepublication history for this paper is available online To view these files, please visit the journal online (http://dx.doi org/10.1136/bmjopen-2018023426).

Received 6 April 2018 Revised 8 August 2018 Accepted 9 August 2018

Check for updates

(c) Author(s) (or their employer(s)) 2018. Re-use permitted under CC BY-NC. No commercial re-use. See rights and permissions. Published by BMJ.

${ }^{1}$ Department for Health, University of Bath, Bath, UK ${ }^{2}$ Severn School of Surgery, Health Education England, Bristol, UK

${ }^{3}$ Department of Trauma and Orthopaedics, Bristol Royal Hospital for Children, Bristol, UK ${ }^{4}$ Somerset Spinal Surgery Service, Musgrove Park Hospital Taunton and Somerset NHS Foundation Trust, Taunton, UK

Correspondence to Mr James W A Fletcher; jwa.fletcher@doctors.net.uk

\section{ABSTRACT}

Introduction There are major differences between legal and medical approaches to informed consent. Medically, consent is obtained prospectively for an intended procedure, to inform the patient of choices, risks and benefits, and to manage expectations. Legally, consent is reviewed retrospectively, usually following unmet expectations and/or the occurrence of complications. Recent legal cases relating to clinical negligence define the establishment of causation and breach of duty related to informed consent. However, there is no prospective evidence to validate the current judicial perspectives on causation and thus clinical negligence. The aim of this randomised controlled trial (RCT) is to investigate whether variations in consenting processes for the same procedure lead to changes in patient decision-making related to consent for that procedure.

Methods and analysis The Risks In Spinal Consenting for Surgery trial is a single-centre, non-inferiority RCT, where 220 patients, aged over 18 years, receiving an elective, day case spinal injection, will be randomised to either a 'legally styled' consent form with 55 risks identified in the world literature, or a 'medically styled' consent form with the 13 serious or most common risks usually quoted by reference to specialist society guidelines. Following explanation of the medical reasons for considering an injection therapy and consent to the trial, participants will be randomly allocated to one of two groups (1:1). The patients are then given the opportunity to discuss any concerns relating to the procedure and/ or risks with a single specialist practitioner. The primary outcome will be rates of consent withdrawal due to the risks explained. Secondary outcomes include scores from the State-Trait Anxiety Inventory, Visual Analogue Scale, EuroQol 5-dimension questionnaire and 0swestry Disability Index.

Ethics and dissemination Results will be presented in peer-reviewed journals and at international conferences. This study is approved by the Health Research Authority: REC 16/SC/0510.

Trial registration number ISRCTN67513618; Pre-results.

\section{Strengths and limitations of this study}

- This is the first study attempting to prospectively assess patient decision-making when randomised to different explanations of the risks in a consent process.

- This study provides a methodology of how to compare different consent processes for the same procedure.

- Measuring anxiety scores will provide an assessment of potential negative consequences of either process.

- Spinal injections are a relatively minor procedure, so results may not be generalisable to more major procedures, though conversely participants may be more likely to withdraw consent for a minor than a major procedure, and the risks explained still include potentially serious conditions.

- No participant blinding is possible given the types of intervention; they will know which style of consent form that they have.

\section{INTRODUCTION}

Patient decision-making when consenting for surgery has been extensively tested in court. ${ }^{1}$ Patients have been found legally correct, when stating post hoc, that they may not have given consent if certain risks had been presented to them preoperatively. Explaining risks associated with any procedure is beneficial for ethical, medical and legal reasons. Ethically, it is better for the patient and the surgeon to follow a shared decision-making process regarding proceeding to an operation. Medically, a patient should be aware of their potential immediate, early and late health statuses after an intervention. Legally, consent is required to waive liability should recognised and anticipated unavoidable 
complications arise or patient expectations not be met. These aspects are relevant to all consenting procedures worldwide.

The risks material to a procedure have previously been dictated by the treating surgeon and, if needed, their peers, under the Bolam principle of practice. ${ }^{2}$ However, this stance has been deemed incorrect by the recent Montgomery judgement, ${ }^{3}$ which judges any risk that would be thought material in a patient's opinion should be discussed. However, once it has occurred, any complication can be retrospectively considered as a material risk by the patient. ${ }^{3}$ The Montgomery judgement also makes comment on the information process, saying it is insufficient to 'bombard' patients with large volumes of information simply to waive risk of litigation. The combination of these factors has changed medical negligence outcomes considerably over recent years. This is despite there being no clinical evidence to support the legal view that patient decision-making will often materially change based on the preoperative risks presented to them. This has led to a shift in how surgeons approach the consent process. The classical 'medical-styled' consent process aimed to focus the patient on pertinent risks of an operative procedure. We feel the current clinical negligence climate only supports surgeons who adopt a 'legal-styled' approach which presents the patient with an encyclopaedic list of potential operative risks.

The aim of this randomised controlled trial (RCT) is to investigate whether different consenting processes for the same procedure actually lead to changes in granting consent for that procedure.

\section{METHODS AND ANALYSIS \\ Study design}

This study protocol describes the design of this singlecentre, non-inferiority RCT. The study protocol conforms with the Standard Protocol Items: Recommendations for Interventional trials ${ }^{4}$ The study will be reported to conform with the Consolidated Standards of Reporting Trials ${ }^{5}$ statement for reporting an RCT. Patients will be recruited from the Somerset Spinal Surgery Service of Musgrove Park Hospital, Taunton and Somerset National Health Service (NHS) Foundation Trust, Taunton, UK. The study is registered at ISRCTN67513618 ${ }^{6}$; enrolment started in May 2017 and is scheduled to finish in March 2018, with the trial completing in April 2019.

\section{Patients}

Two hundred and twenty patients fulfilling the eligibility criteria will be included:

\section{Inclusion criteria}

- Able to consent independently.

- Pre-existing psychiatric conditions including anxiety will not be excluded.

- Age over 18 years.
- Diagnostic and/or treatment injections to the cervical, thoracic, lumbosacral spine, coccyx and sacroiliac joints.

- Facet joint blocks/nerve root and dorsal root ganglion injections/caudal epidural/transforaminal epidural.

\section{Exclusion criteria}

- Patients listed for inpatient procedure.

- Emergency injections.

- Patients who are unable to understand English will be excluded because the questionnaires in this study have not been translated and validated into all other languages.

- Patients who lose capacity before they receive their injection.

\section{Recruitment procedure}

The trial recruitment flow is outlined in figure 1 and participant timeline in figure 2.

Patients reviewed in Spinal Surgery Service clinics at Musgrove Park Hospital, who meet the eligibility criteria, will be invited to participate in the trial. Patients will have been referred to clinic by triage physiotherapists, other orthopaedic surgeons, general practitioners (GPs) or may be seen as a routine follow-up. Patients will be offered a spinal injection as part of their diagnostic and/ or therapeutic management. The reason for suggesting treatment with an injection will be explained by the clinician. Patients will then be asked to consider participation in the trial, explaining that currently it is unclear what effect giving information about potential risks during the consenting process has on the decisions made by patients, and what anxiety, if any, it may cause. Patients will verbally consent to consider the trial in clinic and be given an information pack (Pack A). Pack A will contain a patient information sheet about the trial and a consent form for the trial alongside a stamped addressed envelope (SAE), with no information regarding the injection itself. This will give patients time to reflect on the aim of the trial, whether they want to participate and whether they want the injection offered to them. The trial consent form also provides the patient an opportunity to decline trial participation but still proceed with the injection or reject the injection entirely.

Participants will be instructed to return the pack A 'trial' consent form in the SAE. On receipt of this, they will be sent a randomised consent form with its respective risks detailed, State-Trait Anxiety Inventory (STAI) state and trait questionnaires and an SAE, all contained in pack B. These packs will be randomised, placed in a tray and sent out in a sequential order by the spinal secretaries. Patients receive pack $\mathrm{B}$ in a randomised 1:1 allocated fashion. This ensures patients receiving pack $\mathrm{B}$ are not subject to sampling bias, that is, declining entry in to the trial based purely on the consent form they have been randomised to receive. We have used a computer-generated randomisation schedule to allocate patients to receive either a medical-styled or legal-styled consent form as part of 
Patient seen in spinal clinic and offered spinal injection and participation in trial

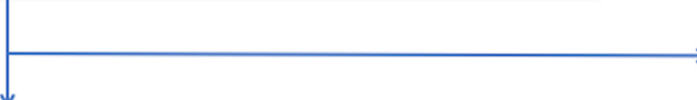

Patient verbally agrees to trial and is given 'Pack A' containing: patient information leaflet, trial checklist and Stamped Addressed Envelope (SAE) in trial information pack
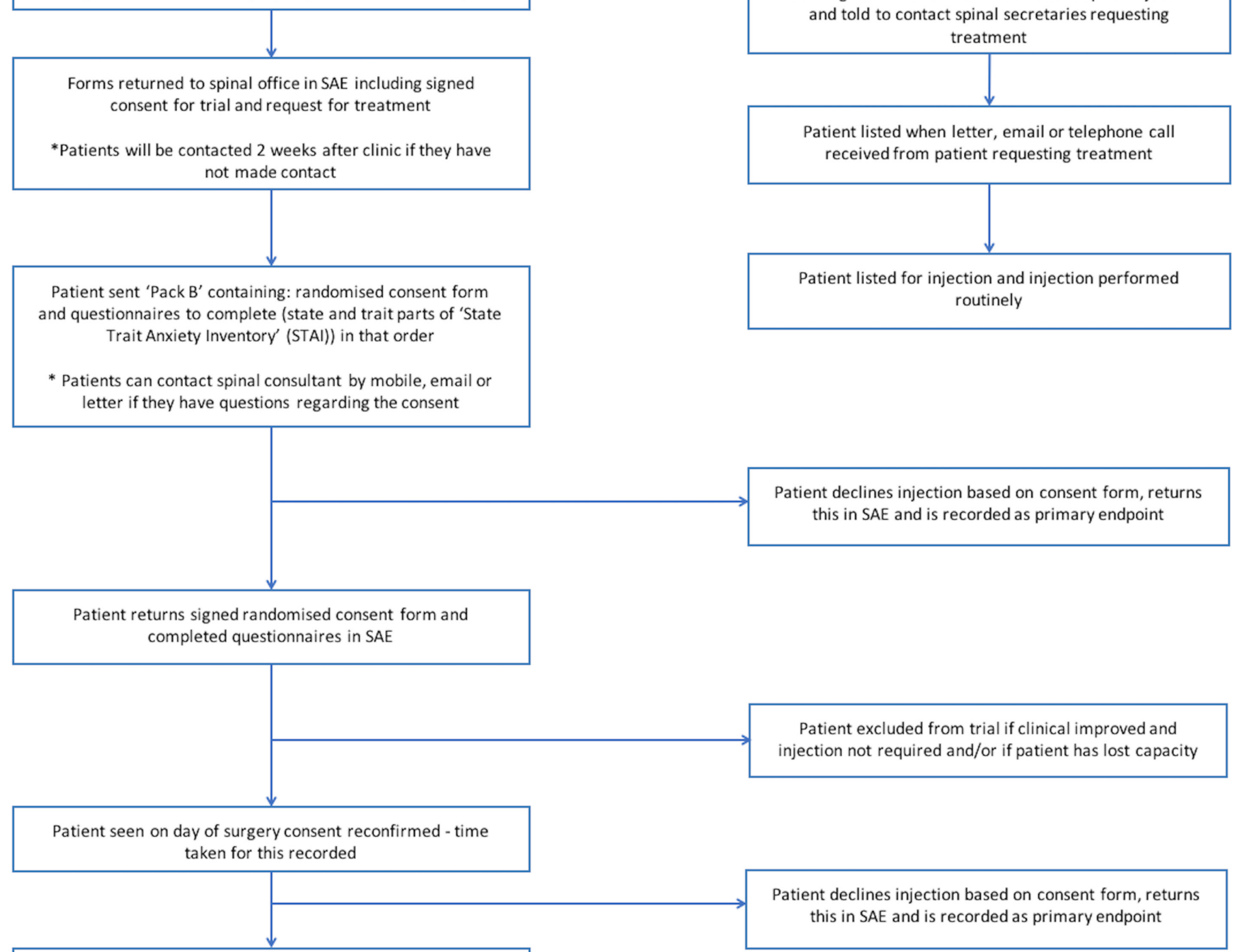

Patient continues to consent - physiological parameters and STAI state remeasured

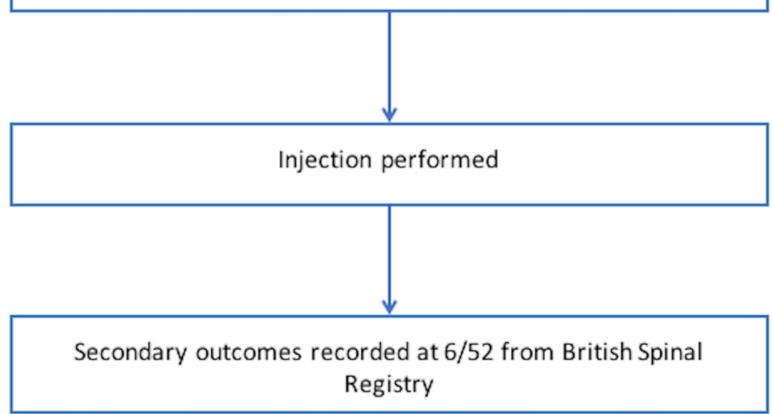

Figure 1 Flow chart of participant journey.
Patient declines to participate in trial

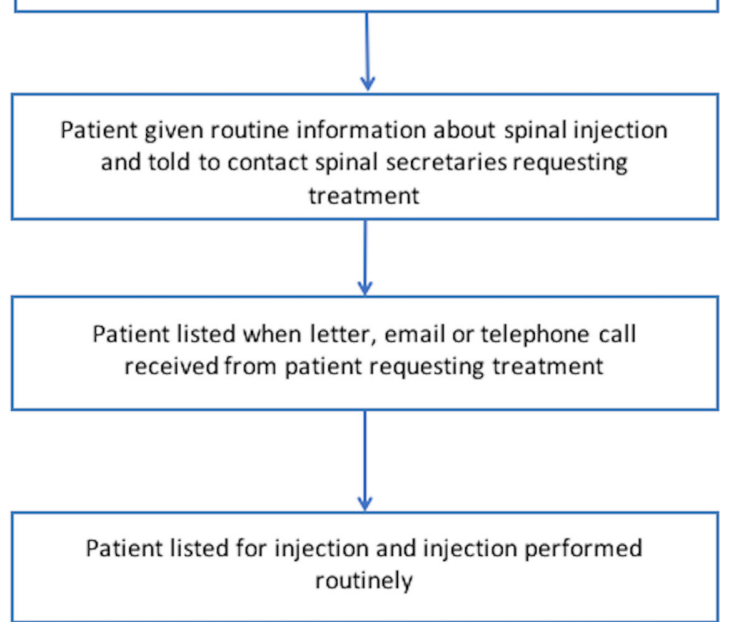

theclines injection based on consent form, returns s in SAE and is recorded as primary endpoint

Patient excluded from trial if clinical improved an njection not required and/or if patient has lost capacity 


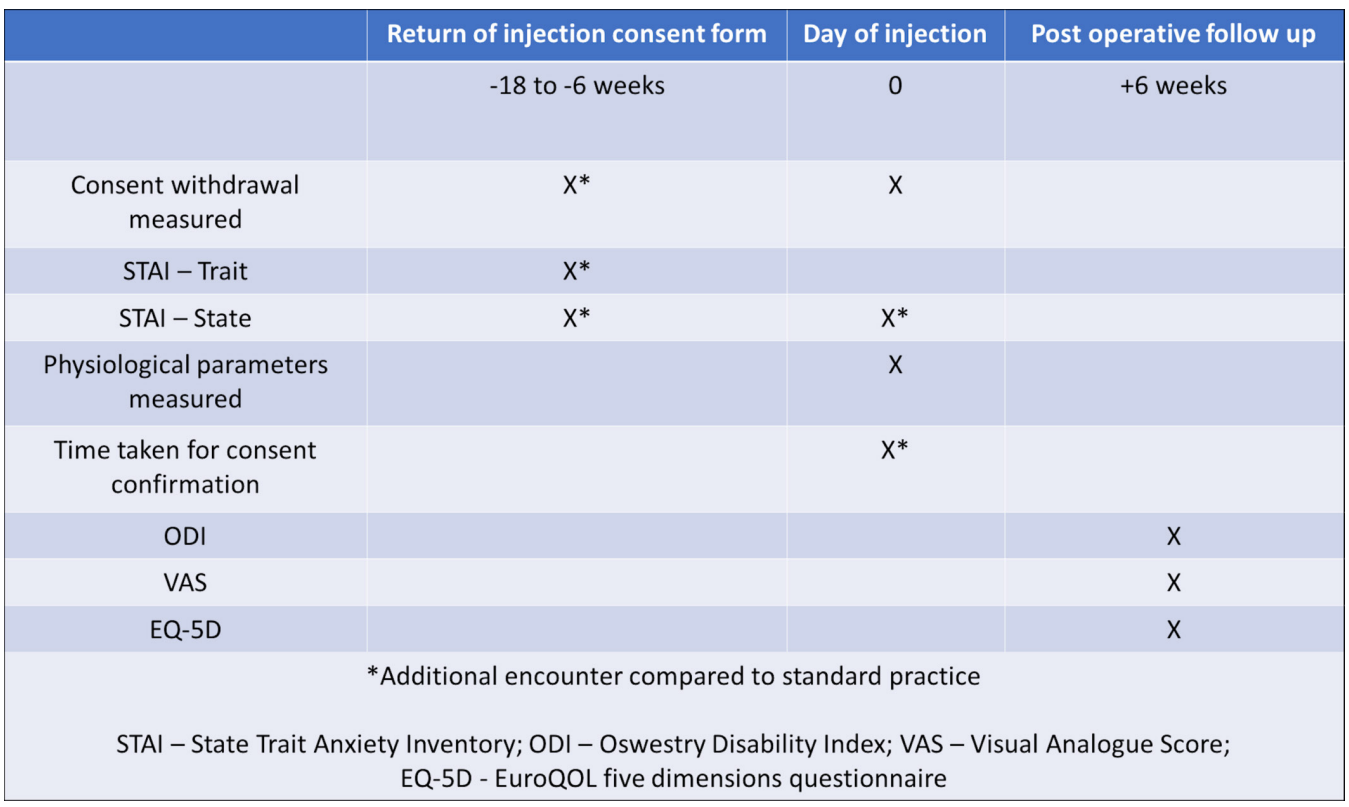

Figure 2 RISCS trial participant encounters. RISCS, Risks In Spinal Consenting for Surgery.

their pack B. The envelopes containing the forms will be in a box, ordered as per the randomisation sequence of four to six patients. The surgeons and spinal secretaries administering the trial packs will be blinded to the randomisation order. There will be a contact number included to allow patients to discuss any concerns, or have certain risks explained in further detail. This explanation will be undertaken by a single clinician, to avoid variations in the explanations of specific risks.

Participants will be asked to read the consent form including the detailed risks. They will also complete the STAI questionnaires as part of pack B. If any participant has any questions about the procedure or the trial, they will have the contact details of the chief investigator (a consultant spinal surgeon) and will be encouraged to contact them via 24 hours mobile, email or letter. Having reviewed and signed their consent form, participants will then complete their anxiety questionnaires (STAI). There will be an SAE contained in this pack, allowing them to return their consent form and questionnaires.

There will be a follow-up telephone call from the spinal secretaries after 2 weeks if the forms have not been received; patients will be asked to allow for their telephone number (confirmed at their clinical appointment) to be used to communicate with them for the trial if needed. Once received, the consent forms will be filed in the participant's notes, and the questionnaires and trial consent forms will be anonymised and stored securely in the trial $\log$ held in the spinal office.

Patients who withdraw from treatment following receipt of the consent form will be contacted by the chief investigator to ascertain the reason for withdrawal, specifically improvement in symptoms or concern with the risks of the procedure.

On the day of surgery, consent will be reconfirmed by the treating clinician. This will involve ensuring that the participant still has symptoms, understands the planned procedure and risks, and has signed the procedure consent form. Following this, a STAI-state questionnaire will be assessed alongside physiological measures to identify if there is any change in anxiety with the consent reconfirmation process or related to the admission itself. This will be performed for both intervention and control groups. The time taken for confirmation of consenting will be measured and used as a marker of the extra time taken to explain the additional risks on the intervention consent form.

The participant will then have their spinal injection. There will be no further active participant interactions required for the trial. Secondary outcome measures related to patient-reported outcome measures (PROMs) will be recorded from the British Spinal Registry (BSR) ${ }^{7}$ that all patients undergoing procedures in the Somerset Spinal Surgery Service are allocated to.

\section{Intervention}

Participants will have either the standard consent form or the intervention form. Both forms will be identical except for the risks that are mentioned. Current practice for injection treatments is for consenting in clinic or on the day of surgery. This will be changed to have consent reconfirmed on the day of surgery, with the consent form having been signed and returned by the patient in advance. This will give patients adequate time to make an informed decision regarding their treatment.

The standard risks ('medically styled') that a patient is informed of during the consenting process are:

Drug reactions (transient flushing, rash/itching); sensory/motor block, failure to improve symptoms; pain; dural tear; allergic reaction; bleeding; stroke; wrong level/site; nerve injury; cauda equina injury; soft-tissue infection. These are based on the complications on the 
British Association of Spinal Surgeons' (BASS) registry website, the BSR.

The intervention consent form ('legally styled') will be encyclopaedic to include all known risks and complications to have ever have occurred from spinal injections following a detailed literature search:

Drug reactions (transient flushing, rash/itching); sensory/motor block, failure to improve symptoms; pain; dural tear; allergic reaction; bleeding; stroke $^{89}$; wrong level/site; nerve injury; cauda equina injury ${ }^{10}$; softtissue infection, haematoma formation, damage to adjacent structures (pneumothorax (if thoracic injection) ${ }^{11}$ /bladder and/or bowel injury (if lumbar/caudal epidural)), cerebellar herniation, ${ }^{12}$ risk of steroids (transient decrease in immunity, high blood sugars, ${ }^{13}$ stomach ulcers, avascular necrosis, cataracts, increased appetite, menstrual irregularities, nausea, diarrhoea, euphoria, depression, local fat atrophy, increased risk of spinal fracture, increased temperature ${ }^{14}$; skin discoloration; spinal headache ${ }^{15}$; vascular injury ${ }^{16}$; $\operatorname{arachnoiditis}^{17}$; paralysis (paraplegia, ${ }^{18}$ quadriplegia $^{19}{ }^{20}$ ); meningeal irritation; intradural/epidural/subdural abscess ${ }^{21-23}$; septic arthritis of facet joint ${ }^{24}$; disc infection ${ }^{24}$; meningitis ${ }^{22}$; CSF-cutaneous fistula ${ }^{25}$; retinal haemorrhage ${ }^{26}$; prolonged blockade $^{27}$; intravascular injection ${ }^{28} 29$; conus medullaris syndrome ${ }^{30}$; brain thrombophlebitis ${ }^{31}$; spinal cord infarction $^{32}{ }^{33}$; cortical blindness $^{34}$; seizures ${ }^{35}$; brain oedema; death. ${ }^{1236}$

\section{DATA COLLECTION \\ Outcomes}

Primary outcome: withdrawal of consent due to risks

Withdrawal of consent due to the risks stated will be recorded as the primary outcome measure. Withdrawal of consent can occur at any time after inclusion in the trial. If the patient withdraws from treatment due to improvement in their symptoms and thus does not consent, then they will be excluded from the data analysis. If the participant had given written consent and returned their consent form and subsequently declined treatment due to an improvement in symptoms, they would be excluded from the analysis.

\section{Secondary outcomes}

\section{The State-Trait Anxiety Inventory}

The STAI questionnaire ${ }^{37}$ has two parts to it, assessing the current state of anxiety and the anxiety trait of the patient. Both parts will be completed by the patient at home, with only the state part needing to be reassessed at the time of reconfirmation of consent on the day of surgery. The STAI is one of the most widely used subjective measures of anxiety in health research. It contains two 20-item self-report scales designed to measure how much worry, tension or apprehension the subject experiences in his or her present circumstances (state anxiety) and how much anxiety represents a personality characteristic (trait anxiety). Items emphasise the frequency of particular symptoms (ranging from $1=$ not at all to $4=$ verymuch so). A minimal important difference of 10 has been used in another study. ${ }^{38}$ Form $\mathrm{Y}$ will be used in this study as it has a more replicable factor structure and improved psychometric properties. ${ }^{39}$

\section{Visual Analogue Scale}

Visual Analogue Scales are used routinely as PROMs postoperatively and will be recorded in the BSR database. This has been shown to be a reliable, valid and responsive to changes in pain $^{40}$ and will be recorded from the BSR at 6 weeks postoperatively.

\section{EuroQol 5-dimension}

The EuroQol 5-dimension measures five dimensions on a three-point scale: mobility, self-care, daily activities, pain/ discomfort, anxiety/depression; no, some or extreme problems. A utility score can be calculated to reflect the valuation of that health state in a society, in this case using the UK tariff. ${ }^{41}$ These scores are routinely recorded in the BSR database. These will be accessed at 6 weeks postoperatively.

\section{Oswestry Disability Index}

The Oswestry Disability Index is used in spinal procedures to quantify symptomatic changes pre-interventions and post-interventions and how the back or leg pain affects the patient's everyday life. ${ }^{42}$ It has 10 questions each with six possible answers, with each answer receiving a score between 0 and 5 , yielding a score ranging between 0 and 50 (which is scaled to $100 \%$ ). These are routinely recorded in the BSR database and will be accessed at 6 weeks postoperatively.

\section{Physiological measures}

Baseline physiological measures (heart rate, respiratory rate, blood pressure) will be recorded before and immediately after confirmation of consent on the day of surgery.

\section{Time for confirmation of consent}

The time taken for the risks to be explained and questions answered will be recorded on the day of surgery.

\section{Recruitment rate}

Approximately 20-30 injections occur as a day case each week at the trial hospital. Based on 10 injections a week (33\%-50\% recruitment rate), 22 weeks will be needed to recruit patients. There will be up to an 18-week waiting time from listing to injection due to NHS waiting lists (figure 2). This will allow the patient to have time to reflect on their decisions regarding inclusion in the trial and their treatment.

Some patients' symptoms will improve while waiting for their injection or they may develop more pressing medical issues that take priority. In either case, patients may withdraw from having their injection on medical grounds. This is anticipated to be up to $15 \%$ of patients, and the recruitment calculations reflect this. 


\section{Follow-up}

Final follow-up from the trial will be at 6 weeks postinjection as part of their routine spinal follow-up. There will be remote follow-up of PROMS using the BSR database. Patients' data will be analysed on an intention-to-treat analysis, though as choosing not to consent is the primary outcome measure, there will be no crossover between the groups.

\section{Statistical considerations}

Given that the background to the intervention is that it is thought to not affect the rates of consent, it can be assessed as a non-inferior treatment. The primary outcome measure is binary.

For a non-inferiority trial, at 5\% significance, $90 \%$ power, assuming that $99.5 \%$ of patients do not withdraw their consent when the risks are explained normally (eg, 199 patients out of 200 consent), to show that there is a $3 \%$ difference in the rates of consent, 95 patients are needed per group (that would be 95 consenting in one group and less than 92 out of 95 in the other to show difference). If there is truly no difference between the standard and experimental treatment, then 190 patients are required to be $90 \%$ sure that the upper limit of a one-sided $95 \%$ CI will exclude a difference in favour of the standard group of more than 3\%. Anticipating $15 \%$ drop-out due to improvement in symptoms and/or more pressing medical issues, 110 patients will be recruited per group.

\section{Data management}

Data will be collected by surgeons and the spinal research team at the trial hospital. This will be stored securely on trust computers within the spinal office with data entry and coding of the de-identified data conducted by trained staff. The final data set will be accessible to the chief investigator and stored for 5 years following the end of the study.

\section{Statistical analysis}

Data will be analysed using IBM SPSS Statistics for Windows, V.20 (IBM Corp). Independent t-test will be used for analysis within the groups tested and MannWhitney $\mathrm{U}$ tests to compare the intervention and control groups. Data analysis will be performed by statisticians blinded to the intervention.

\section{Patient public involvement}

Patients who have had spinal injections have helped design the methodology regarding the timings and number of forms to complete. The reading level of the checklist form has been measured as Flesch-Kincaid Reading Ease 74.6 (100 being the easiest), with the most complicated form explaining the risks in more detail still being of a general public reading level (Flesch-Kincaid Reading Ease 59.5). ${ }^{41}$

\section{ETHICS AND DISSEMINATION}

This study will be conducted in agreement with the Declaration of Helsinki. The questionnaires have been approved by the Clinical Research Support Department at Musgrove Park Hospital prior to their distribution and will be used under their appropriate licence.

Standard practice (the control group) will be improved as the control consent form will be based on the national guidance from the BASS. Current legal (though not NHS nor BASS) guidance would state that the intervention consent form has neither ethical implications nor harmful effects to the patient as a consent form with a complete list of the risks, that a patient may deem material, should be being used. By measuring psychological and physiological stress, if harm is caused by more extensive consent forms, this can be identified. If the rates of consent withdrawal are seen to be statistically significant at an early analysis point (after 50 patients), then the trial would be stopped early. If any patients are found to be significantly anxious on review of the completed questionnaires, they will be offered referral to their GP for onward management of their anxiety.

Patients will be provided with Patient Advice Liaison Service contact information, should they want to talk to someone independent about the trial (information is on the patient information leaflet).

Patients will be provided with the contact details of the chief investigator so that they can raise any questions regarding the study or their injection. A list of any patients who use this service, and those who make any contact with the Somerset Spinal Surgery Service via other means (eg, telephone to secretaries, email to spinalsurgeryservice@tst.nhs.uk) and the reason for this contact will be recorded; all patient encounters are already contemporaneously logged on the hospital electronic patient record system.

\section{Dissemination}

Results will be submitted for publication in an international, peer-reviewed journal regardless of the outcomes. Additionally, findings will be presented at local, regional and international ethical, orthopaedic and spinal conferences.

\section{POTENTIAL OUTCOMES}

This work is unique in its concept. There is currently no objective and prospective evidence to support the legally enshrined principle that giving more information alters the rates of consent in patients; the Risks In Spinal Consenting for Surgery (RISCS) trial addresses this. If rates of consent do decrease with more information, especially regarding rare risks, then the legal principle is upheld and all consenting practice in the NHS should change to reflect this. This would often involve significant change in practice, mainly relating to the time allocated to consent processes and the amount of information imparted; also, the time given to patients to reflect on this information. Conversely, if there is no change in the rates of consent despite more detailed explanation of risks, then the premise of the Chester versus Afshar 
Supreme Court judgement will be shown to be fallible, and this study may be used to justify and defend standard consenting practice for minor procedures. Further to this, this study may show that it is harmful to attempt to explain all risks to patients, in that it creates physiological disturbances and psychological stress as shown by the STAI questionnaires. This would further justify that standard explanation of risk and consenting is appropriate. While directly relevant to UK law, the findings will have transferability to practices worldwide given the consistency in the aspects that underpin consent processes. Finally, following the completion of the RISCS trial, the methodology will be used to design a further trial investigating causality using more major procedures (RISCS 2) to investigate whether the principal outcome holds for all procedures.

Contributors JWAF, MK and PT wrote the protocol and designed the study; all authors critically reviewed the manuscript.

Funding This study was supported by the Bristol Orthopaedic Trust and the Henry Bissell Spinal Fund.

Competing interests None declared.

Patient consent Not required.

Ethics approval This study is approved by the Health Research Authority: REC 16/ SC/0510 and will be conducted in agreement with the Declaration of Helsinki.

Provenance and peer review Not commissioned; externally peer reviewed.

Open access This is an open access article distributed in accordance with the Creative Commons Attribution Non Commercial (CC BY-NC 4.0) license, which permits others to distribute, remix, adapt, build upon this work non-commercially, and license their derivative works on different terms, provided the original work is properly cited, appropriate credit is given, any changes made indicated, and the use is non-commercial. See: http://creativecommons.org/licenses/by-nc/4.0/.

\section{REFERENCES}

1. Chester v Afshar [2004] UKHL 41.

2. Bolam v Friern Barnet Hospital Management Committee [1957]. 1 WLR 582. 2 All ER 118 (QBD).

3. Montgomery v Lanarkshire Health Board [2015]. UKSC 11. All ER (D) 113 (Mar).

4. Chan AW, Tetzlaff JM, Altman DG, et al. SPIRIT 2013 statement: defining standard protocol items for clinical trials. Ann Intern Med 2013;158:200-7.

5. Moher D, Hopewell S, Schulz KF, et al. CONSORT 2010 explanation and elaboration: updated guidelines for reporting parallel group randomised trials. BMJ 2010;340:c869.

6. Fletcher J, Khan M, Thorpe P. RISCS Trial - Risks In Spinal Consenting for Surgery: establishing the information required to enable informed consent for patients receiving spinal injections, 2017. ISRCTN67513618.

7. BASS. British Spinal Registry Online, 2017. (accessed 01/05/2017).

8. Ziai WC, Ardelt AA, Llinas RH. Brainstem stroke following uncomplicated cervical epidural steroid injection. Arch Neurol 2006;63:1643-6.

9. Linn AJ, Desilva C, Peeters-Asdourian C. Thromboembolic stroke: a rare complication associated with peri-procedural management of an epidural steroid injection. Pain Physician 2009;12:159-62.

10. Bilir A, Gulec S. Cauda equina syndrome after epidural steroid injection: a case report. J Manipulative Physiol Ther 2006;29:492. e1-3.

11. Zaugg M, Stoehr S, Weder W, et al. Accidental pleural puncture by a thoracic epidural catheter. Anaesthesia 1998;53:69-71.

12. Beckman WA, Mendez RJ, Paine GF, et al. Cerebellar herniation after cervical transforaminal epidural injection. Reg Anesth Pain Med 2006;31:282-5.
13. Benoist M, Boulu P, Hayem G. Epidural steroid injections in the management of low-back pain with radiculopathy: an update of their efficacy and safety. Eur Spine J 2012;21:204-13.

14. Committee JF. British national formulary 73. London: BMJ Publishing and the Royal Pharmaceutical Society, 2017. (accessed 3 Mar 2017).

15. Webb CA, Weyker PD, Zhang L, et al. Unintentional dural puncture with a Tuohy needle increases risk of chronic headache. Anesth Analg 2012;115:124-32.

16. Karasek M, Bogduk N. Temporary neurologic deficit after cervical transforaminal injection of local anesthetic. Pain Med 2004;5:202-5.

17. Koerts G, Rooijakkers H, Abu-Serieh B, et al. Postoperative spinal adhesive arachnoiditis presenting with hydrocephalus and cauda equina syndrome. Clin Neurol Neurosurg 2008;110:171-5.

18. Kennedy DJ, Dreyfuss $P$, Aprill CN, et al. Paraplegia following imageguided transforaminal lumbar spine epidural steroid injection: two case reports. Pain Med 2009;10:1389-94.

19. Bose B. Quadriparesis following cervical epidural steroid injections: case report and review of the literature. Spine J 2005;5:558-63.

20. Tripathi M, Nath SS, Gupta RK. Paraplegia after intracord injection during attempted epidural steroid injection in an awake-patient. Anesth Analg 2005;101:1209-11.

21. Zimmerer SM, Conen A, Müller AA, et al. Spinal epidural abscess: aetiology, predisponent factors and clinical outcomes in a 4-year prospective study. Eur Spine J 2011;20:2228-34.

22. Hooten WM, Kinney MO, Huntoon MA. Epidural abscess and meningitis after epidural corticosteroid injection. Mayo Clin Proc 2004;79:682-6.

23. Saigal G, Donovan Post MJ, Kozic D. Thoracic intradural Aspergillus abscess formation following epidural steroid injection. AJNR Am J Neuroradiol 2004;25:642-4.

24. Weingarten TN, Hooten WM, Huntoon MA. Septic facet joint arthritis after a corticosteroid facet injection. Pain Med 2006;7:52-6.

25. Tsai A, Ahmed S, Wang J. Persistent cerebrospinal fluid-cutaneous fistula after epidural analgesia: a case report and review of literature. journal of Anesthesiology and Clinical Science 2014;3:2.

26. Kushner $\mathrm{FH}$, Olson JC. Retinal hemorrhage as a consequence of epidural steroid injection. Arch Ophthalmol 1995;113:309.

27. Arndt JA, Downey T. Exceptionally prolonged anesthesia after a small dose of intrathecal bupivacaine. Anesthesiology 2002;97:1042-42.

28. Manchikanti L, Malla Y, Wargo BW, et al. Complications of fluoroscopically directed facet joint nerve blocks: a prospective evaluation of 7,500 episodes with 43,000 nerve blocks. Pain Physician 2012;15:E143-50.

29. Nahm FS, Lee CJ, Lee SH, et al. Risk of intravascular injection in transforaminal epidural injections. Anaesthesia 2010;65:917-21.

30. Tackla RD, Keller JT, Ernst RJ, et al. Conus medullaris syndrome after epidural steroid injection: Case report. Int J Spine Surg 2012;6:29-33.

31. Kate MP, Thomas B, Sylaja PN. Cerebral venous thrombosis in postlumbar puncture intracranial hypotension: case report and review of literature. F1000Res 2014;3:41.

32. Ludwig MA, Burns SP. Spinal cord infarction following cervical transforaminal epidural injection: a case report. Spine 2005;30:E266-E268.

33. Somayaji HS, Saifuddin A, Casey AT, et al. Spinal cord infarction following therapeutic computed tomography-guided left $L 2$ nerve root injection. Spine 2005;30:E106-E108.

34. Young WF. Transient blindness after lumbar epidural steroid injection: a case report and literature review. Spine 2002;27:E476-7.

35. Landa J, Kim Y. Outcomes of interlaminar and transforminal spinal injections. Bull NYU Hosp Jt Dis 2012;70:6-10.

36. Stauber B, Ma L, Nazari R. Cardiopulmonary arrest following cervical epidural injection. Pain Physician 2012;15:147-52.

37. Spielberger CD, Gorsuch RL, Lushene R, et al. Manual for the Statetrait anxiety inventory. Palo Alto, CA: Consulting psychologists press Inc, 1983

38. Corsaletti BF. Minimal important difference for anxiety and depression surveys after intervention to increase daily physical activity in smokers. Fisioterapia e Pesquisa 2014;21:359-64.

39. Oei TP, Evans L, Crook GM. Utility and validity of the STAI with anxiety disorder patients. Br J Clin Psychol 1990;29(Pt 4):429-32.

40. Carlsson AM. Assessment of chronic pain. I. Aspects of the reliability and validity of the visual analogue scale. Pain 1983;16:87-101.

41. Devlin NJ, Shah KK, Feng Y, et al. Valuing health-related quality of life: An EQ-5D-5L value set for England. Health Econ 2018;27:7-22.

42. Fairbank JC, Couper J, Davies JB, et al. The Oswestry low back pain disability questionnaire. Physiotherapy 1980;66:271-3. 